\title{
Long Time Behavior for the Equation of Finite-Depth Fluids
}

\section{Guo Boling, Tan Shaobin ${ }^{\star}$}

Institute of Applied Physics and Computational Mathematics, P.O. Box 8009, Beijing 100088, P. R. China

Received: 1 March 1993

Abstract: In this paper we study the Cauchy problem for the generalized equation of finite-depth fluids

$$
\partial_{t} u-G\left(\partial_{x}^{2} u\right)-\partial_{x}\left(\frac{u^{p}}{p}\right)=0,
$$

where $G(\cdot)$ is a singular integral, and $p$ is an integer larger than 1 . We obtain the long time behavior of the fundamental solution of linear problem, and prove that the solutions of the nonlinear problem with small initial data for $p>5 / 2+\sqrt{21} / 2$ are decay in time and freely asymptotic to solutions of the linear problem. In addition we also study some properties of the singular integral $G(\cdot)$ in $L^{q}(R)$ with $q>1$.

\section{Introduction}

In this paper we shall consider the Cauchy problem for the generalized equation of finite-depth fluids

$$
\partial_{t} u-G\left(\partial_{x}^{2} u\right)+\partial\left(\frac{u^{p}}{p}\right)=0,
$$

where $p$ is an integer larger than $1, G(f)=\lim _{\varepsilon \rightarrow 0} \int_{|y| \geqq \varepsilon>0} f(x-y) K(y) d y$ with $K(y)=\frac{1}{2 \delta}\left(\operatorname{coth} \frac{\pi y}{2 \delta}-\operatorname{sign} y\right)$ is a singular integral, here $\delta$ is a positive real which characterizes the depth of the fluid layer. Equation (1) was first derived by Joseph $[5,10]$ to describe the propagation of internal waves in the stratified fluid of finite depth. It is known [1] that Eq. (1) reduces to the nonlinear Korteweg-de Vries $(\mathrm{KdV})$ equation

$$
\partial_{t} u-\partial_{x}^{3} u+\partial\left(\frac{u^{p}}{p}\right)=0
$$

\footnotetext{
$\star$ Present address: Department of Mathematics and Statistics, University of Saskatchewan, Saskatoon, S7N OWO, Canada
} 
and the Benjamin-Ono (BO) equation

$$
\partial_{t} u-H\left(\partial_{x}^{2} u\right)+\partial\left(\frac{u^{p}}{p}\right)=0
$$

for $p=2$ as the depth $\delta$ tends respectively to zero and infinity, where $H(\cdot)$ denotes the Hilbert transform. From the view point of mathematics, there is an amount of work devoted to studying large time behavior problem for the solutions of the nonlinear $\mathrm{KdV}$ equation and $\mathrm{BO}$ equation (see $[3,6,7,11,12]$ and the references therein). In particular, if we denote by $U(\cdot)$ and $V(\cdot)$ the free evolution group which solve respectively the Cauchy problems of linear $\mathrm{KdV}$ equation $\partial_{t} u-\partial_{x}^{3} u=0$, and the linear BO equation $\partial_{t} u-H\left(\partial_{x}^{2} u\right)=0$, then one has the following available results:

$$
\|U(t) f\|_{p} \leqq C t^{-\frac{1}{3}\left(1-\frac{2}{p}\right)}\|f\|_{p^{\prime}}
$$

and

$$
\|V(t) f\|_{p} \leqq C t^{-\frac{1}{2}\left(1-\frac{2}{p}\right)}\|f\|_{p^{\prime}}
$$

for all $t \geqq 1$, and $p \geqq 2, p^{\prime}=p /(p-1)$. In the present paper we shall obtain a similar decay estimate for the fundamental solution of the linear problem

$$
\partial_{t} u-G\left(\partial_{x}^{2} u\right)=0, \quad u(x, 0)=f(x)
$$

i.e. we gave

$$
\|u\|_{p} \leqq C\left(t^{-\frac{1}{2}\left(1-\frac{2}{p}\right)}+(\delta t)^{-\frac{1}{3}\left(1-\frac{2}{p}\right)}\right)\|f\|_{p^{\prime}}
$$

for all $t \geqq 1$. Furthermore, we shall substitute the decay estimate (4) into the integral equation associated with the nonlinear equation (1) to obtain long time behavior for the nonlinear problem (1). However, as we shall see, because of the complication of the symbol $P(\xi)$, which characterizes the dispersive relation of Eq. (1), it is much more difficult to prove the decay estimate (4) for the linear problem (3), and requires more a elaborate calculation than to obtain the estimates (2) for the linear $\mathrm{KdV}$ equation and linear $\mathrm{BO}$ equation. In fact the dispersive relation $P(\xi)$ for Eq. (1) is $(2 \pi \xi)^{2}\left(\operatorname{coth}(2 \pi \delta \xi)-\frac{1}{2 \pi \delta \xi}\right)$, while for nonlinear $\mathrm{KdV}$ equation and $\mathrm{BO}$ equation the dispersive relations are respectively the simple forms $\xi^{3}$ and $|\xi| \xi$.

This paper is organized as follows. In Sect. 2 we state some basic lemmas, and give certain properties for the singular integral $G(\cdot)$. In Sect. 3 we consider the fundamental solution of the linear problem (3). The decay estimate (4) is proved by applying the Van der Corput Lemma [14]. Finally in Sect. 4 we exploit the results of Sects. 2 and 3 to derive the time decay estimate and free asympotic property for the nonlinear problem (1).

To conclude this section, we give the main notation used in this paper. By $C$ we denote various positive constants which may be different from line to line, and is independent of time $t$ and the functions to be estimated. For all $p, 1 \leqq p \leqq \infty$, we denote by $\|\cdot\|_{p}$ the norm in $L^{p}(R)$. 


\section{Preliminary Results}

We denote by $\hat{f}$ and $\breve{f}$ the Fourier transform and its inversion for function $f(x)$, i.e.

$$
\hat{f}(\xi)=\int_{R} f(x) e^{-2 \pi i \xi x} d x, \check{f}(x)=\int_{R} f(\xi) e^{2 \pi i \xi x} d \xi .
$$

For the singular integral $G(\cdot)$, we can derive the following properties

Lemma 1. For any function $f(x), g(x) \in C_{0}^{\infty}(R)$, we have
a. $[G(f)]^{\wedge}=-i\left[\operatorname{coth}(2 \pi \delta \xi)-\frac{1}{2 \pi \delta \xi}\right] \hat{f}$,
b. $G\left(\partial_{x} f\right)=\partial_{x} G(f)$,
c. $\int_{R} f G(g) d x=-\int_{R} g G(f) d x$,
d. $\int_{R} f_{x}\left(G\left(f_{x}\right)\right)^{2} d x+\frac{2}{\delta} \int_{R} f f_{x} G\left(f_{x}\right) d x=\frac{1}{3} \int_{R}\left(f_{x}\right)^{3} d x$,
e. $\|G(f)\|_{2} \leqq\|f\|_{2}$.

Proof. See Zhou Yulin, et al. [17].

The above lemma shows that the singular integral $G(\cdot)$ is a linear bounded transform from Hilbert space $H^{s}(R)$ into itself for $s \geqq 0$. It is to be noted that the inequality (e) in fact can be extended to the case of a function taking value in Sobolev space $L^{p}(R)$, for $1<p<\infty$.

Lemma 2. For any function $f \in L^{p}(R), 1<p<\infty$, we have

$$
\|G(f)\|_{p} \leqq C(p)\|f\|_{p},
$$

where the positive constant $C$ is independent of $f$.

In order to prove Lemma 2, we need the following results.

Lemma 3. Suppose the kernel $K(x)$ satisfies the following conditions:

$$
\begin{gathered}
|K(x)| \leqq B|x|^{-1}, \quad \text { for }|x|>0, \\
\int_{R_{1}<|x|<R_{2}} K(x) d x=0, \quad \text { for } 0<R_{1}<R_{2}<\infty, \\
\int_{|x| \geqq 2|y|}|K(x-y)-K(x)| d x \leqq B, \quad \text { for }|y|>0,
\end{gathered}
$$

where $B$ is a positive constant. Then for any $f \in L^{p}(R), 1<p<\infty$, we have

$$
\|T(f)\|_{p} \leqq C(p)\|f\|_{p},
$$

where $T(f)(x)=\lim _{\varepsilon \rightarrow 0} \int_{|y| \geqq \varepsilon>0} f(x-y) K(y) d y$.

Proof. See E.M. Stein [13]

Lemma 4. For $|y| \neq 0$, we have

$$
y^{2} \operatorname{coth} y=y+\frac{1}{3} y^{3}-2 y^{3} \sum_{k=1}^{\infty} \frac{y^{2}}{k^{2} \pi^{2}\left(k^{2} \pi^{2}+y^{2}\right)},
$$




$$
y^{2} \operatorname{coth} y=y|y|\left(1+2 \sum_{k=1}^{\infty} e^{-2 k|y|}\right) \text {. }
$$

Proof. The first expansion (8) was given in [1]. Here we need only to show the second expansion of $y^{2} \operatorname{coth} y$. In fact, for $y>0$,

$$
\begin{aligned}
y^{2} \operatorname{coth} y & =y^{2} \frac{e^{y}+e^{-y}}{e^{y}-e^{-y}}=y^{2}\left(1+\frac{2 e^{-2 y}}{1-e^{-2 y}}\right) \\
& =y^{2}\left(1+2 e^{-2 y} \sum_{k=0}^{\infty} e^{-2 k y}\right)=y^{2}\left(1+2 \sum_{k=1}^{\infty} e^{-2 k y}\right) .
\end{aligned}
$$

And for $y<0$ we can obtain

$$
y^{2} \operatorname{coth} y=-y^{2}\left(1+2 \sum_{k=1}^{\infty} e^{2 k y}\right) .
$$

Therefore, we obtain (9).

Now we are in a position to prove Lemma 2. On account of Lemma 3, we need only to check that the kernel $K(x)=\frac{1}{2 \delta}\left(\operatorname{coth} \frac{\pi y}{2 \delta}-\operatorname{sign} y\right)$ satisfies the conditions (5)-(7).

Proof of Lemma 2. First, for $|x| \neq 0$, By using lemma 4, we see that

$$
K(x)=\delta^{-1} \operatorname{sign} x \sum_{k=1}^{\infty} e^{-\delta^{-1} \pi k|x|}
$$

This gives us

$$
|x K(x)|=\delta^{-1}|x| \sum_{k=1}^{\infty} e^{-\delta^{-1} \pi k|x|}=\frac{\delta^{-1}|x|}{e^{\delta^{-1} \pi|x|}-1} \leqq \frac{1}{\pi} .
$$

Next, for $0<R_{1}<R_{2}<\infty$, one can easily check (6) by using the expansion (9).

Finally, we prove condition (7). For $|y| \neq 0$, we have

$$
\begin{aligned}
\int_{|x| \geqq 2|y|}|K(x-y)-K(x)| d x= & \delta^{-1} \int_{|x| \geqq 2|y|} \mid \operatorname{sign}(x-y) \sum_{k=1}^{\infty} e^{-k \delta^{-1} \pi|x-y|} \\
& \quad-\operatorname{sign} x \sum_{k=1}^{\infty} e^{-k \delta^{-1} \pi|x|} \mid d x \\
= & \delta^{-1} \sum_{k=1}^{\infty}\left[\int_{2|y|}^{\infty}\left|e^{-k \delta^{-1} \pi(x-y)}-e^{-k \delta^{-1} \pi x}\right| d x\right. \\
& \left.+\int_{2|y|}^{\infty}\left|e^{-k \delta^{-1} \pi x}-e^{-k \delta^{-1} \pi(x+y)}\right| d x\right] \\
= & I(y) .
\end{aligned}
$$

We now split the proof into two cases. First, for $y>0$, we have 


$$
\begin{aligned}
I(y)= & \delta^{-1} \sum_{k=1}^{\infty} \int_{2 y}^{\infty} e^{-k \delta^{-1} \pi x} d x\left(e^{k \delta^{-1} \pi y}-e^{-k \delta^{-1} \pi y}\right) \\
& =\frac{1}{\pi} \sum_{k=1}^{\infty} \frac{1}{k}\left(e^{-k \delta^{-1} \pi y}-e^{-3 k \delta^{-1} \pi y}\right) \\
& =\frac{1}{\pi} \ln \frac{\left(e^{3 \delta^{-1} \pi y}-1\right) e^{\delta^{-1} \pi y}}{e^{3 \delta^{-1} \pi y}\left(e^{\delta^{-1}} \pi y-1\right)} \leqq \frac{3}{\pi}
\end{aligned}
$$

By a similar manner, we can also see that the inequality (10) valid for $y<0$. The proof of Lemma 2 is then complete.

Lemma 5. Let $\phi(y)=x y+y^{2} \operatorname{coth} y$, then for $|y| \neq 0$, we have $\phi^{\prime}(y)=\phi^{\prime}(-y)$, $\left|\phi^{\prime \prime}(y)\right|=\phi^{\prime \prime}(|y|)$. Moreover, for $|y|>2$, we have $\left|\phi^{\prime \prime}(y)\right|>2$.

Proof. From Lemma 4, we can easily deduce that

$$
\begin{aligned}
& \phi^{\prime}(y)=x+2|y|+4 \sum_{k=1}^{\infty}\left(|y|-k y^{2}\right) e^{-2 k|y|}, \\
& \phi^{\prime \prime}(y)=\operatorname{sign} y\left(2+8 \sum_{k=1}^{\infty}\left(y^{2} k^{2}-2|y| k+\frac{1}{2}\right) e^{-2 k|y|}\right),
\end{aligned}
$$

and

$$
\phi^{\prime \prime}(y)=2 y-2 y \sum_{k=1}^{\infty} \frac{20 k^{4} \pi^{4} y^{2}+18 k^{2} \pi^{2} y^{4}+6 y^{6}}{k^{2} \pi^{2}\left(k^{2} \pi^{2}+y^{2}\right)^{3}}
$$

Furthermore if we set $h(y)=\frac{20 a^{2} y+18 a y^{2}+6 y^{3}}{(a+y)^{3}}$, for $a>0, y>0$, then we have

$$
h^{\prime}(y)=\frac{4 a^{2}(5 a-y)}{(a+y)^{4}} .
$$

Therefore, the results of the lemma can be derived simply by using (11)-(14).

Lemma 6. For any function $f \in C_{0}^{\infty}(R)$, we have

$$
\int_{R} f G\left(\partial_{x} f\right) d x \geqq A\|f\|_{H^{\frac{1}{2}(R)}}-B\|f\|_{2},
$$

where the positive constants $A$ and $B$ are independent of $f$.

Proof. On account of Lemma 1, and by using the Parseval identity

$$
\begin{aligned}
\int_{R} f G\left(\partial_{x} f\right) d x & =\int_{R} 2 \pi \xi\left(\operatorname{coth}(2 \pi \delta \xi)-\frac{1}{2 \pi \delta \xi}\right)|\hat{f}|^{2} d \xi \\
& =2 \pi\left(\int_{|\xi|>k_{0}}+\int_{|\xi| \leqq k_{0}}\right)
\end{aligned}
$$

where $k_{0}$ is a positive number to be chosen later.

Since $\lim _{|\xi| \rightarrow \infty}\left(\operatorname{coth}(2 \pi \delta \xi)-\frac{1}{2 \pi \delta \xi}\right)=\operatorname{sign}(\xi)$, thus there exists a sufficient large constant $k_{0}>0$, such that, for all $|\xi|>k_{0}$, the first integral in (15) satisfies 


$$
2 \pi \int_{|\xi|>k_{0}} \xi\left(\operatorname{coth}(2 \pi \delta \xi)-\frac{1}{2 \pi \delta \xi}\right)|\hat{f}|^{2} d \xi \geqq \pi \int_{|\xi|>k_{0}}|\xi||\hat{f}|^{2} d \xi .
$$

Moreover, in order to handle the second integral in (15), we apply Lemma 4,

$$
\begin{aligned}
& 2 \pi \int_{|\xi| \leqq k_{0}} \xi\left(\operatorname{coth}(2 \pi \delta \xi)-\frac{1}{2 \pi \delta \xi}\right)|\hat{f}|^{2} d \xi \\
& =\int_{|\xi| \leqq k_{0}} \xi^{2}\left(\frac{4 \pi^{2} \delta}{3}-8 \delta \sum_{n=1}^{\infty} \frac{4 \delta^{2} \xi^{2}}{n^{2}\left(n^{2}+4 \delta^{2} \xi^{2}\right)}\right)|\hat{f}|^{2} d \xi \\
& \geqq \int_{|\xi| \geqq k_{0}} \xi^{2}\left(\frac{4 \pi^{2} \delta}{3}-8 \delta \sum_{n=1}^{\infty} \frac{4 \delta^{2} k_{0}^{2}}{n^{2}\left(n^{2}+4 \delta^{2} k_{0}^{2}\right)}\right)|\hat{f}|^{2} d \xi \\
& =\frac{4 \pi^{2} \delta}{3} \varepsilon_{0} \int_{|\xi| \leqq k_{0}} \xi^{2}|\hat{f}|^{2} d \xi
\end{aligned}
$$

where we have used the identity: $\sum_{k=1}^{\infty} \frac{1}{n^{2}}=\frac{\pi^{2}}{6}$, and the $\varepsilon_{0}$ is a positive constant depending only on $\delta$ and $k_{0}$.

Therefore, from (16), (17) it follows that

$$
\begin{aligned}
& \int_{R} f G\left(\partial_{x} f\right) d x \geqq \pi \int_{|\xi|>k_{0}}|\xi||\hat{f}|^{2} d \xi+\frac{4 \pi^{2} \delta \varepsilon_{0}}{3} \int_{|\xi| \leqq k_{0}} \xi^{2}|\hat{f}|^{2} d \xi \\
& =\pi \int_{|\xi|>k_{0}}|\xi||\hat{f}|^{2} d \xi+\frac{4 \pi^{2} \delta \varepsilon_{0}}{3} \int_{|\xi| \leqq k_{0}}\left(1+\xi^{2}\right)|\hat{f}|^{2} d \xi-\frac{4 \pi^{2} \delta \varepsilon_{0}}{3} \int_{|\xi| \leqq k_{0}}|\hat{f}|^{2} d \xi \\
& \geqq A \int_{R}(1+|\xi|)|\hat{f}|^{2} d \xi-B \int_{R}|\xi|^{2} d \xi \\
& =A\|f\|_{H^{1 / 2}(R)}-B\|f\|_{2},
\end{aligned}
$$

where $A=\min \left\{\pi, 8 \pi^{2} \delta \varepsilon_{0} / 3\right\}, B=A+4 \pi^{2} \delta \varepsilon_{0} / 3$.

\section{Linear Estimates}

In this section we shall obtain the decay estimate concerning the fundamental solution of the linear problem

$$
\begin{gathered}
\partial_{t} u-G\left(\partial_{x}^{2} u\right)=f, \\
u(x, 0)=u_{0}(x) .
\end{gathered}
$$

Define the unitary group $W(t) u_{0}=g_{t} * u_{0}$, where

$$
g_{t}(x)=\int_{R} e^{i(2 \pi \xi)^{2}\left(\operatorname{coth}(2 \pi \delta \xi)-\frac{1}{2 \pi \delta \xi}\right) t+2 \pi i \xi x} d \xi,
$$

then the solution of (18), (19) can be written as

$$
u(x, t)=W(t) u_{0}(x)+\int_{0}^{t} W(t-s) f(x, s) d s .
$$


Our purpose in this section is to estimate the oscillatory integral $g_{t}(x)$ for all $t \geqq 0$.

Lemma 7. Let $\delta>0$ be a constant, then

$$
\left\|g_{t}(x)\right\|_{\infty} \leqq C\left(t^{-1 / 2}+(\delta t)^{-1 / 3}\right)
$$

for all $t \geqq 1$, where the constant $C$ is independent of $t$ and $\delta$.

Let $\eta=2 \pi \delta \xi, \tau=\delta^{-2} t$, and $y=\frac{x}{\tau \delta}-1$, then $g_{t}(x)$ can be rewritten as

$$
S_{\tau}(y)=2 \pi \delta g_{t}(x)=\int_{R} e^{i \tau\left(\eta^{2} \operatorname{coth} \eta+y \eta\right)} d \eta
$$

In order to bound $S_{\tau}(y)$ for all $y \in R$, we need the following classical Van der Corput lemma [14]

Lemma 8. Suppose that $\psi \in C_{0}^{1}(R)$ and $\phi \in C^{2}(R)$ such that $\left|\phi^{\prime \prime}(\xi)\right| \geqq 1$ on the support of $\psi$. Then

$$
\left|\int_{R} e^{i \lambda \phi(\xi)} \psi(\xi) d \xi\right| \leqq C \lambda^{-1 / 2}\left\{\|\psi\|_{\infty}+\left\|\psi^{\prime}\right\|_{1}\right\},
$$

where the constant $C$ is independent of $\lambda, \phi$ and $\psi$. The prime denotes the derivative.

Proof of Lemma 7. We write $S_{t}(x)$ as

$$
S_{t}(x)=\int_{|\eta| \leqq 3} e^{i t \phi(\eta)} d \eta+\int_{|\eta|>3} e^{i t \phi(\eta)} d \eta=S_{t}^{1}(x)+S_{t}^{2}(x),
$$

where $\phi(\eta)=x \eta+\eta^{2} \operatorname{coth} \eta$.

We shall split the proof into two parts to bound respectively $S_{1}(x)$ and $S_{2}(x)$ for all $x \in R$.

Part 1. Taking the variable-transform $\xi=t^{1 / 3} \eta, \quad y=t^{2 / 3}(1+x)$, and applying Lemma 4, we have

$$
S_{t}^{1}(x)=t^{-1 / 3} \int_{|\xi| \leqq 2 t^{1 / 3}} e^{i \bar{\phi}(\xi)} d \xi
$$

where $\bar{\phi}(\xi)=y \xi+\frac{1}{3} \xi^{3}-2 \xi^{3} \sum_{k=1}^{\infty} \frac{\left(t^{-1 / 3} \xi\right)^{2}}{\pi^{2} k^{2}\left(\pi^{2} k^{2}+\left(t^{-1 / 3} \xi\right)^{2}\right)}$.

In what follows, we shall show that the identity

$$
\int_{|\xi| \leqq 3 t^{1 / 3}} e^{i \bar{\phi}(\xi)} d \xi
$$

is bounded uniformly for all $t \geqq 0, y \in R$.

In fact, one can easily see that the boundedness of $(25)$ is equivalent with the boundedness of the following integral for all $t \geqq 1$ and $y \in R$ :

$$
\int_{R} e^{i \bar{\phi}(\xi)} \bar{\varphi}(\xi) d \xi
$$

where the cut-off function $\bar{\varphi}(\xi) \in C^{\infty}(R)$, satisfies $0 \leqq \bar{\varphi} \leqq 1$, and $\bar{\varphi} \equiv 1$ for $2 \leqq|\xi| \leqq$ $3 t^{1 / 3}, \bar{\varphi} \equiv 0$ for $|\xi|<1$ and $|\xi|>3 t^{1 / 3}+1$, and such that $|\bar{\varphi}(\xi)| \leqq C$. 
On account of Lemma 8, the boundedness of (26) can be immediately obtained by checking the positive upper estimate of $\left|\bar{\phi}^{\prime \prime}(\xi)\right|$ in the support of $\bar{\varphi}(\xi)$, i.e. $\left|\bar{\phi}^{\prime \prime}(\xi)\right| \geqq \lambda_{0}$, for all $\xi \in \operatorname{supp}\{\bar{\varphi}(\xi)\}$, and $\lambda_{0}$ is a positive constant. In fact, by using Lemma 5 ,

$$
\begin{aligned}
\left|\bar{\phi}^{\prime \prime}(\xi)\right| & =t^{1 / 3}\left|\phi^{\prime \prime}(\eta)\right| \\
& \geqq 2|\xi|\left(1-\sum_{k=1}^{\infty} \frac{20 k^{4} \pi^{4}\left(t^{-1 / 3} \xi\right)^{2}+18 k^{2} \pi^{2}\left(t^{-1 / 3} \xi\right)^{4}+6\left(t^{-1 / 3} \xi\right)^{6}}{k^{2} \pi^{2}\left(k^{2} \pi^{2}+\left(t^{-1 / 3} \xi\right)^{2}\right)^{3}}\right) \\
& \geqq 2\left(1-\sum_{k=1}^{\infty} \frac{20 k^{4} \pi^{4} \cdot 16+18 k^{2} \pi^{2}(16)^{2}+6(16)^{2}}{k^{2} \pi^{2}\left(k^{2} \pi^{2}+16\right)^{3}}\right) \\
& =\lambda_{0}>2\left(1-\sum_{k=1}^{\infty} \frac{6}{k^{2} \pi^{2}}\right)=0,
\end{aligned}
$$

for all $\xi \in \operatorname{supp}\{\bar{\varphi}(\xi)\}$, and $t \geqq 1$. Therefore, we obtain

$$
\left|S_{t}^{1}(x)\right| \leqq C t^{-1 / 3} \text {, }
$$

for $t>0, x \in R$.

Part 2. In order to bound the integral

$$
S_{t}^{2}(x)=\int_{|\eta|>3} e^{i t \phi(\eta)} d \eta, \quad\left(\phi(\eta)=x \eta+\eta^{2} \operatorname{coth} \eta\right)
$$

for $t>0, x \in R$. We set a cut-off function $\varphi_{0}(\eta) \in C^{\infty}(R)$, which satisfies $0 \leqq$ $\varphi_{0}(\eta) \leqq 1, \varphi_{0}(\eta) \equiv 1$ for $|\eta|>3, \varphi_{0}(\eta) \equiv 0$ for all $|\eta|<3-\varepsilon$, and such that

$$
\left|\partial_{\eta}^{k} \varphi_{0}(\eta)\right| \leqq C(k) \varepsilon^{-k}
$$

for $k=1$, where $\varepsilon \in(0,1)$ is a number to be chosen later. Therefore

$$
\begin{aligned}
\left|S_{t}^{2}(x)\right| & =\left|\int_{R} e^{i t \phi(\eta)} \varphi_{0}(\eta) d \eta-\int_{|\eta| \leqq 3} e^{i t \phi(\eta)} \varphi_{0}(\eta) d \eta\right| \\
& \leqq\left|\int_{R} e^{i t \phi(\eta)} \varphi_{0}(\eta) d \eta\right|+2 \varepsilon .
\end{aligned}
$$

In what follows we shall split the proof into two cases to bound the integral in (28).

Case 1. Assume that $x>-\left(3-8 \sum_{k=1}^{\infty}(2 k-1) e^{-4 k}\right)$. Then from Lemma 5 we can verify that $\left|\phi^{\prime \prime}(\eta)\right|=\phi^{\prime \prime}(|\eta|)>2$, and $\phi^{\prime}(\eta)=\phi^{\prime}(|\eta|)>\phi^{\prime}(2)>1$ for all $|\eta|>2$. Therefore

$$
\begin{aligned}
\left|\int_{R} e^{i t \phi(\eta)} \varphi_{0}(\eta) d \eta\right| \leqq & \frac{1}{t}\left|\int_{R} e^{i t \phi(\eta)} \frac{\varphi_{0}^{\prime}(\eta)}{\phi^{\prime}(\eta)} d \eta\right|+\frac{1}{t} \int_{R} \frac{\left|\varphi_{0}(\eta) \phi^{\prime \prime}(\eta)\right|}{\left(\phi^{\prime}(\eta)\right)^{2}} d \eta \\
\leqq & C t^{-3 / 2}\left(\left\|\frac{\varphi_{0}^{\prime}(\eta)}{\phi^{\prime}(\eta)}\right\|_{\infty}+\left\|\left(\frac{\varphi_{0}^{\prime}(\eta)}{\phi^{\prime}(\eta)}\right)^{\prime}\right\| \|_{1}\right)+t^{-1}\left\|\frac{\varphi_{0}(\eta) \phi^{\prime \prime}(\eta)}{\left(\phi^{\prime}(\eta)\right)^{2}}\right\|_{1} \\
\leqq & C t^{-3 / 2}\left(\left\|\varphi_{0}^{\prime}(\eta)\right\|_{\infty}+\left\|\varphi_{0}^{\prime \prime}(\eta)\right\|_{\infty} \operatorname{mes}\{3-\varepsilon<|\eta|<3\}\right. \\
& \left.+\left\|\varphi_{0}^{\prime}(\eta)\right\|_{\infty} \int_{3-\varepsilon<|\eta|<3} \frac{\left|\phi^{\prime \prime}(\eta)\right|}{\left(\phi^{\prime}(\eta)\right)^{2}} d \eta\right)+t^{-1} \int_{|\eta|>3-\varepsilon} \frac{\left|\phi^{\prime \prime}(\eta)\right|}{\left(\phi^{\prime}(\eta)\right)^{2}} d \eta \\
\leqq & C\left(t^{-1}+\varepsilon^{-1} t^{-3 / 2}\right),
\end{aligned}
$$


where we have used integration by part, and Lemma 8 .

Case 2. Assume that $x \leqq-\left(3-8 \sum_{k=1}^{\infty}(2 k-1) e^{-4 k}\right)$. We note that $-\left(3-8 \sum_{k=1}^{\infty}(2 k-1) e^{-4 k}\right)=-\left(3-\frac{8\left(e^{4}+1\right)}{\left(e^{4}-1\right)^{2}}\right)=-x_{0}<-2$. Therefore, in order to bound the integral $S_{t}^{2}(x)$ in this case, we consider two domains

$$
\begin{aligned}
& \Omega_{1}=\left\{\eta ;\left|\phi^{\prime}(\eta)\right| \leqq|x| / 2\right\}, \\
& \Omega_{2}=\left\{\eta ;\left|\phi^{\prime}(\eta)\right| \leqq|x| / 3\right\},
\end{aligned}
$$

where $\phi^{\prime}(\eta)=\left(x \eta+\eta^{2} \operatorname{coth} \eta\right)^{\prime}=x+2|\eta|+4 \sum_{k=1}^{\infty}\left(|\eta|-k \eta^{2}\right) e^{-2 k|\eta|}$.

The proof in this case is based on the version of the following three lemmas concerning the two domains $\Omega_{1}$ and $\Omega_{2}$.

Lemma 9. Let $x \leqq-x_{0}$, then there exists a constant $\delta_{0}>0$ such that $\{\eta ;|\eta|<$ $\left.\delta_{0}\right\} \subset\left(\bar{\Omega}_{1}\right)^{c}$.

Proof. Since

$$
\phi^{\prime}(\eta)=\left(\eta^{2} \operatorname{coth} \eta+x \eta\right)^{\prime}=x+2 \eta+\frac{4 \eta}{e^{2 \eta}-1}-\frac{4 \eta^{2} e^{2 \eta}}{\left(e^{2 \eta}-1\right)^{2}},
$$

and

$$
\lim _{|\eta| \rightarrow 0}\left(2 \eta+\frac{4 \eta}{e^{2 \eta}-1}-\frac{4 \eta^{2} e^{2 \eta}}{\left(e^{2 \eta}-1\right)^{2}}\right)=1,
$$

thus there exists a constant $\delta_{0}>0$ such that, for all $|\eta|<\delta_{0}$, we have

$$
\left|\phi^{\prime}(\eta)\right|>|x|-\left|2 \eta+\frac{4 \eta}{e^{2 \eta}-1}-\frac{4 \eta^{2} e^{2 \eta}}{\left(e^{2 \eta}-1\right)^{2}}\right|>|x|-x_{0} / 2 \geqq|x| / 2 .
$$

This implies the result of the lemma.

Lemma 10. There exists a constant $C_{0}$, such that

$$
\operatorname{mes}\left\{\Omega_{1} \cap\{|\eta|>2\}\right\} \leqq|x|+C_{0}
$$

for $x \leqq-x_{0}$.

Proof. From (30), we have

$$
\begin{aligned}
\Omega_{1} \cap\{|\eta|>2\} & =\left\{\eta ; \frac{|x|}{4}-2 \sum_{k=1}^{\infty}\left(k \eta^{2}-|\eta|\right) e^{-2 k|\eta|}\right. \\
& \leqq|\eta| \leqq \frac{3|x|}{4}+2 \sum_{k=1}^{\infty}\left(k \eta^{2}-|\eta|\right) e^{-2 k|\eta|} \\
& \text { and }|\eta|>2\} .
\end{aligned}
$$

This gives us 


$$
\begin{aligned}
\operatorname{mes}\left\{\Omega_{1} \cap\{|\eta|>2\}\right\} & \leqq 2 \sup _{|\eta|>2}\left\{\frac{|x|}{2}+4 \sum_{k=1}^{\infty}\left(k \eta^{2}-|\eta|\right) e^{-2 k|\eta|}\right\} \\
& =\left.2\left\{\frac{|x|}{2}+\sum_{k=1}^{\infty}\left(k \eta^{2}-|\eta|\right) e^{-2 k|\eta|}\right\}\right|_{|\eta|=2}=|x|+C_{0} .
\end{aligned}
$$

Lemma 11. Let $r_{0}=\operatorname{dist}\left\{\Omega_{2},\left(\bar{\Omega}_{1}\right)^{c}\right\}$, then there exists a positive constant $C_{1}=$ $C_{1}\left(\delta_{0}\right)$ such that $r_{0}>C_{1}|x|$ for $x \leqq-x_{0}$.

Proof. For any $\xi_{1} \in\left(\bar{\Omega}_{1} \cup\left\{|\eta|<\delta_{0}\right\}\right)^{c}$, and $\xi_{2} \in \Omega_{2}$, we have $\left|\phi^{\prime}\left(\xi_{1}\right)\right|>|x| / 2$, $\left|\phi^{\prime}\left(\xi_{2}\right)\right| \leqq|x| / 3$, which gives us $\left|\phi^{\prime}\left(\xi_{1}\right)-\phi^{\prime}\left(\xi_{2}\right)\right|>|x| / 6$, and

$$
r=\left|\xi_{1}-\xi_{2}\right|>\frac{|x|}{6}\left|\phi^{\prime \prime}\left(\xi_{0}\right)\right|^{-1},
$$

where the point $\xi_{0}$ is on the line connecting $\xi_{1}$ with $\xi_{2}$. Therefore

$$
r_{0}=\inf _{\xi_{1} \in\left(\bar{\Omega}_{1}\right)^{c} ; \xi_{2} \in \Omega_{2}}\left|\xi_{1}-\xi_{2}\right| \geqq \frac{|x|}{6} \inf _{|\xi|>\delta_{0}}\left|\phi^{\prime \prime}(\xi)\right|^{-1}=\frac{|x|}{6} \frac{1}{\sup _{|\xi|>\delta_{0}}\left|\phi^{\prime \prime}(\xi)\right|},
$$

where

$$
\sup _{|\xi|>\delta_{0}}\left|\phi^{\prime \prime}(\xi)\right| \leqq \sup _{|\xi|>\delta_{0}}\left(2+8 \sum_{k=1}^{\infty}\left(\xi^{2} k^{2}+2|\xi| k+1 / 2\right) e^{-2 k|\xi|}\right)=C\left(\delta_{0}\right)>0,
$$

and which finishes the proof of the lemma.

Now we are in a position to consider Case 2. On account of the above three lemmas, we construct a unitary decomposition of $R$, namely we expect two functions such that $\varphi_{1}(\eta), \varphi_{2}(\eta) \in C^{\infty}(R), 0 \leqq \varphi_{1} \leqq 1,0 \leqq \varphi_{2} \leqq 1$, and $\varphi_{1}(\eta)+\varphi_{2}(\eta) \equiv 1$ for all $\eta \in R$. In addition, supp $\left\{\varphi_{1}(\eta)\right\}$ is contained in $\Omega_{1}, \varphi_{2}(\eta) \equiv 0$ for all $\eta \in \Omega_{2}$, and

$$
\left|\partial_{\eta}^{k} \varphi_{i}(\eta)\right| \leqq C(k) r_{0}^{-k} \leqq C|x|^{-k}
$$

for $k=1,2$, and all $\eta \in R$.

The integral in (28) can be then written as

$$
\begin{aligned}
\left|\int_{R} e^{i t \phi(\eta)} \varphi_{0}(\eta) d \eta\right| \leqq & \left|\int_{R} e^{i t \phi(\eta)} \varphi_{0}(\eta) \varphi_{1}(\eta) d \eta\right| \\
& +\left|\int_{R} e^{i t \phi(\eta)} \varphi_{0}(\eta) \varphi_{2}(\eta) d \eta\right| .
\end{aligned}
$$

If $\varphi_{2}(\eta) \neq 0$, i.e. $\eta \notin \Omega_{2}$, then we have $\left|\phi^{\prime}(\eta)\right|>\frac{|x|}{3}$. Note that $|x|+4 \sum_{k=1}^{\infty}\left(-k \eta^{2}+\right.$ $|\eta|) e^{-2 k|\eta|}>0$ for all $|\eta|>2$, we can thus obtain

$$
\left|\phi^{\prime}(\eta)\right| \geqq C^{\prime}\left(|x|+2|\eta|+4 \sum_{k=1}^{\infty}\left(-k \eta^{2}+|\eta|\right) e^{-2 k|\eta|}\right) \geqq 4 C^{\prime}
$$

for all $|\eta|>0$ and $\eta \notin \Omega_{2}$, where $C^{\prime}$ is a positive constant.

Therefore, by applying integration by part and Lemma 8 , we have 


$$
\begin{aligned}
\left|\int_{R} e^{i t \phi(\eta)} \varphi_{0}(\eta) \varphi_{2}(\eta) d \eta\right|= & t^{-1}\left|\int_{R} e^{i t \phi(\eta)}\left(\frac{\varphi_{0}(\eta) \varphi_{2}(\eta)}{\phi^{\prime}(\eta)}\right)^{\prime} d \eta\right| \\
\leqq & C t^{-1}\left\{t^{-1 / 2}\left(\left\|\frac{\varphi_{0}^{\prime}(\eta) \varphi_{2}(\eta)}{\phi^{\prime}(\eta)}\right\|_{\infty}+\left\|\left(\frac{\varphi_{0}^{\prime}(\eta) \varphi_{2}(\eta)}{\phi^{\prime}(\eta)}\right)^{\prime}\right\| \|_{1}\right)\right. \\
& +t^{-1 / 2}\left(\left\|\frac{\varphi_{0}(\eta) \varphi_{2}^{\prime}(\eta)}{\phi^{\prime}(\eta)}\right\|_{\infty}+\left\|\left(\frac{\varphi_{0}(\eta) \varphi_{2}^{\prime}(\eta)}{\phi^{\prime}(\eta)}\right)^{\prime}\right\| \|_{1}\right) \\
& \left.+\left\|\frac{\phi^{\prime \prime}(\eta) \varphi_{0}(\eta) \varphi_{2}(\eta)}{\left(\phi^{\prime}(\eta)\right)^{2}}\right\|_{1}\right\} \\
\leqq & C t^{-3 / 2}\left\{\left\|\varphi_{0}^{\prime}(\eta)\right\|_{\infty}\right. \\
& +\left\|\varphi_{2}^{\prime}(\eta)\right\|_{\infty}+\left\|\varphi_{0}^{\prime \prime}(\eta)\right\|_{\infty} \operatorname{mes}\{3-\varepsilon<|\eta|<3\} \\
& +\left\|\varphi_{0}^{\prime}(\eta)\right\|_{\infty}\left\|\varphi_{2}^{\prime}(\eta)\right\|_{\infty} \operatorname{mes}\{3-\varepsilon<|\eta|<3\} \\
& +\left\|\varphi_{2}^{\prime}(\eta)\right\|_{\infty} \operatorname{mes}\left\{\Omega_{1} \cap\{|\eta|>2\}\right\} \\
& +\left\|\varphi_{0}^{\prime}(\eta)\right\| \infty\left\|\frac{\phi^{\prime \prime}(\eta)}{\left(\phi^{\prime}(\eta)\right)^{2}}\right\|_{L^{1}(|\eta|>2)} \\
\leqq & C t^{-3 / 2}\left(\varepsilon^{-1}+C\right)+C t^{-1} . \\
& \left.+\left\|\varphi_{2}^{\prime}(\eta)\right\|_{\infty}\left\|\frac{\phi^{\prime \prime}(\eta)}{\left(\phi^{\prime}(\eta)\right)^{2}}\right\|_{L^{1}(|\eta|>2)}\right\} \\
& +\frac{\phi^{\prime \prime}(\eta)}{\left(\phi^{\prime}(\eta)\right)^{2} \|_{L^{1}(|\eta|>2)}} \\
& +33) \\
&
\end{aligned}
$$

Moreover, if $\eta \in \Omega_{1}$, then

$$
\begin{aligned}
\left|\int_{R} e^{i t \phi(\eta)} \varphi_{0}(\eta) \varphi_{1}(\eta) d \eta\right| \leqq & C t^{-1 / 2}\left(\left\|\varphi_{0}(\eta) \varphi_{1}(\eta)\right\|_{\infty}+\left\|\left(\varphi_{0}(\eta) \varphi_{1}(\eta)\right)^{\prime}\right\|_{1}\right) \\
\leqq & C t^{-1 / 2}\left(1+\left\|\varphi_{0}^{\prime}(\eta)\right\|_{\infty} \operatorname{mes}\{3-\varepsilon<\{\eta \mid<3\}\right. \\
& \left.+\left\|\varphi_{0}^{\prime}(\eta)\right\|_{\infty} \operatorname{mes}\left\{\Omega_{1} \cap\{|\eta|>2\}\right\}\right) \\
\leqq & C t^{-1 / 2}
\end{aligned}
$$

Finally, combining (33), (34) with (28), and taking $\varepsilon=t^{-1 / 2}$, we thus have

$$
\left|S_{t}^{2}(x)\right| \leqq C t^{-1 / 2}
$$

for all $x \in R$ and $t>1$.

Therefore, on account of the transform (22), we then obtain the desired decay estimate of Lemma 7 by combining (27), (35) and (23).

Theorem 1. Suppose that $g_{t}(x)$ is the unitary group generated by the linear problem of Eq. (1). Let $u(x, t)=g_{t}(x) * u_{0}(x)$, then for $p \in(2, \infty)$, 


$$
\|u(x, t)\|_{p} \leqq C\left(t^{\frac{1}{2}\left(1-\frac{2}{q}\right)}+(\delta t)^{-\frac{1}{3}\left(1-\frac{2}{p}\right)}\right)\left\|u_{0}(x)\right\|_{q},
$$

for all $t \geqq 1$, with $p^{-1}+q^{-1}=1$, where the constant $C$ is independent of $t$ and $\delta$. Proof. By using Lemma 7 , and the $L^{2}(R)$-conservation law of the linear problem (3), the result of the theorem then follows from the Riesz-Thorin interpolation.

Remark. Since the constant $C$ in Theorem 1 is independent of $\delta>0$, we thus see that the decay estimate $(2 b)$ for the linear $\mathrm{BO}$ equation is a consequence of Theorem 1.

\section{Decay Estimates for Nonlinear Problem}

In this section we consider the following nonlinear problem:

$$
\begin{gathered}
\partial_{t} u-G\left(\partial_{x}^{2} u\right)=\partial_{x}\left(u^{p} / p\right), \\
u(x, 0)=u_{0}(x),
\end{gathered}
$$

where $p \geqq 1$ is an integer. It is known $[1,17]$ that for any function $u_{0}(x) \in$ $H^{s}(R)(s \geqq 2)$, there exists a positive constant $T$ such that the nonlinear equation (1) with the initial data $u_{0}(x)$ admits a unique solution in $L^{\infty}\left(0, T ; H^{s}(R)\right)$.

Lemma 12. For any initial data $u_{0}(x) \in H^{k}(R)$, then the solution $u=u(x, t)$ of problem (36), (37) such that

$$
\|u\|_{H^{3}} \leqq\left\|u_{0}\right\|_{H^{3}} \exp \left(C \int_{0}^{t}\|u(\tau)\|_{W^{2, q(R)}}^{p-1} d \tau\right),
$$

for $p \geqq 2$.

Proof. Since, for $k>2$,

$$
\begin{aligned}
\frac{d}{d t} \int_{R}\left|\partial_{x}^{k} u\right|^{2} d x= & 2 \int_{R} \partial_{x}^{k} u\left[\partial_{x}^{k}\left(u^{p-1} \partial_{x} u\right)-u^{p-1} \partial_{x}^{k+1} u\right] d x \\
& +2 \int_{R} \partial_{x}^{k} u \partial_{x}^{k+1} u u^{p-1} d x \\
\leqq & C\left\|\partial_{x}^{k} u\right\|_{2}\left(\left\|\partial_{x}\left(u^{p-1}\right)\right\|_{\infty}\left\|\partial_{x}^{k} u\right\|_{2}+\left\|\partial_{x} u\right\|_{\infty}\left\|\partial_{x}^{k}\left(u^{p-1}\right)\right\|_{2}\right) \\
& +(p-1)\left\|\partial_{x} u\right\|_{\infty}\|u\|_{\infty}^{p-2}\left\|\partial_{x}^{k} u\right\|_{2}^{2} \\
\leqq & C\|u\|_{\infty}^{p-2}\left\|\partial_{x} u\right\|_{\infty}\left\|\partial_{x}^{k} u\right\|_{2}^{2} \\
\leqq & C\|u\|_{W^{2, q}(R)}^{p-1}\left\|\partial_{x}^{k} u\right\|_{2}^{2}
\end{aligned}
$$

where we have used the calculus of inequalities [4].

By applying the Gronwall lemma, (39) is then implying the result of the lemma.

Theorem 2. Let $\delta \in(0, \infty), q=2 p$, and $p>5 / 2+\sqrt{21} / 2$. Assume that the initial data $u_{0}(x)$ is sufficiently small in $H^{3}(R) \cap W^{2,2 p /(2 p-1)}(R)$. Then the solution $u$ of nonlinear problem (36), (37) such that 


$$
\|u(t)\|_{W^{2, q}(R)} \leqq C(1+t)^{-\frac{1}{3}\left(1-\frac{2}{q}\right)},
$$

for all $t \geqq 0$, where the constant $C$ is independent of $u$ and $t$.

Proof. Since the nonlinear problem (36), (37) can be written into the following formula:

$$
u(t)=g_{t} * u_{0}+\int_{0}^{t} g_{t-s} * \partial_{x}\left(u^{p}(s) / p\right) d s
$$

By using Theorem 1 and Hölder inequality, we obtain

$$
\begin{aligned}
\|u(t)\|_{W^{2, q}(R)} \leqq & \left\|g_{t} * u_{0}\right\|_{W^{2, q}(R)} \\
& +\int_{0}^{t}\left\|g_{t-s} * \partial_{x}\left(u^{p} / p\right)\right\|_{W^{2, q}(R)} d s \\
\leqq & C(1+t)^{-\frac{1}{3}\left(1-\frac{2}{q}\right)}\left\|u_{0}\right\|_{W^{2, q^{\prime}(R)}}+C \int_{0}^{t}(t-s)^{-\frac{1}{3}\left(1-\frac{2}{q}\right)}\left\|\partial_{x}\left(u^{p} / p\right)\right\|_{W^{2, q^{\prime}(R)}} d s \\
\leqq & C(1+t)^{-\frac{1}{3}\left(1-\frac{2}{q}\right)}\left\|u_{0}\right\|_{W^{2, q^{\prime}}(R)}+C \int_{0}^{t}(t-s)^{-\frac{1}{3}\left(1-\frac{2}{q}\right)}\|u\|_{W^{2, q}(R)}^{p-1}\|u\|_{H^{3}(R)} d s \\
\leqq & C(1+t)^{-\frac{1}{3}\left(1-\frac{2}{q}\right)}\left\|u_{0}\right\|_{W^{2, q^{\prime}(R)}}+C\left\|u_{0}\right\|_{H^{3}} \int_{0}^{t}(t-s)^{-\frac{1}{3}\left(1-\frac{2}{q}\right)}\|u\|_{W^{2, q}(R)}^{p-1} \\
& \cdot \exp \left(C \int_{0}^{s}\|u(\tau)\|_{W^{2, q}(R)}^{p-1} d \tau\right) d s .
\end{aligned}
$$

Let $\quad M(t)=\sup _{0 \leqq s \leqq t}(1+s)^{\frac{1}{3}\left(1-\frac{2}{q}\right)}\|u(s)\|_{W^{2, q}(R)}, \quad$ and $\quad \bar{\delta}=\left\|u_{0}\right\|_{H^{3}(R)}$ $+\left\|u_{0}\right\|_{W^{2,2 p /(2 p-1)(R)}}$. The above inequality then gives us

$$
M(t) \leqq C \bar{\delta}+C \bar{\delta} f(t) M^{p-1}(t) \exp \left(C h(t) M^{p-1}(t)\right)
$$

where

$$
f(t)=(1+t)^{\frac{1}{3}\left(1-\frac{1}{p}\right)} \int_{0}^{t}(t-s)^{-\frac{1}{3}\left(1-\frac{1}{p}\right)}(1+s)^{-\frac{p-1}{3}\left(1-\frac{1}{p}\right)} d s, h(t)=
$$
$\int_{0}^{t}(1+s)^{-\frac{p-1}{3}\left(1-\frac{1}{p}\right)} d s$.

Note that $p>5 / 2+\sqrt{21} / 2$, one can easily check that there exists a constant $C$ such that $f(t) \leqq C$, and $h(t) \leqq C$, for all $t>0$. Therefore, (40) gives us

$$
M(t) \leqq C \bar{\delta}+C \bar{\delta} M^{p-1}(t) \exp \left(C M^{p-1}(t)\right) .
$$

Let $K(m)=c \bar{\delta}\left(1+m^{p-1} \exp \left(C m^{p-1}\right)\right)-m$. Since $K(0)=C \bar{\delta}>0$, and $K^{\prime \prime}(m)$ $>0$, for all $m>0$, we take $\bar{\delta}>0$ sufficiently small so that $K(m)=0$ admits a positive zero $m_{1}$. Then set $C \bar{\delta}<m_{1}$, as $K(M(t)) \geqq 0$ for all $t>0$, and $M(0)=C \bar{\delta}<m_{1}$, so on account of the continuity of $K(M(t))$, we finally obtain that $M(t) \leqq m_{1}$ for all $t \geqq 0$, i.e. $\|u(t)\|_{W^{2, q}(R)} \leqq m_{1}(1+t)^{-\frac{1}{3}\left(1-\frac{2}{q}\right)}$, for all $t \geqq 0$, and $q=2 p>5+\sqrt{21}$.

Theorem 3. Under the conditions of Theorem 2, the solution of the nonlinear problem (36), (37) is freely asymptotic to the solution of linear problem (3). 
Proof. Denoted by $u(t) \in L^{\infty}\left(R^{+} ; H^{3}(R) \cap W^{2,2 p /(2 p-1)}(R)\right)$ the solution of the nonlinear problem (36), (37), we shall prove that there exists a function $u_{+}(t) \in$ $L^{\infty}\left(R^{+} ; H^{2}(R)\right)$, such that

$$
\partial_{t} u_{+}(t)-G\left(\partial_{x}^{2} u_{+}(t)\right)=0
$$

and

$$
\left\|u(t)-u_{+}(t)\right\|_{H^{2}(R)} \rightarrow 0
$$

as $t \rightarrow+\infty$. In fact, from Lemma 12 and Theorem 1 , we have

$$
\|u(t)\|_{H^{3}} \leqq\left\|u_{0}\right\|_{H^{3}} \exp \left(C \int_{0}^{t}\|u(\tau)\|_{W^{2, q}(R)}^{p-1} d \tau\right) \leqq C,
$$

Therefore, we define as in $[7,12]$

$$
u_{+}(t)=u(t)-\int_{t}^{+\infty} g_{t-s} * \partial_{x}\left(u^{p} / p\right)(s) d s .
$$

One can easily find that above function satisfies the linear equation (42), and such that

$$
\begin{aligned}
\left\|u(t)-u_{+}(t)\right\|_{H^{2}} & \leqq C \int_{t}^{+\infty}\|u(s)\|_{W^{2, q}}^{p-1}\|u(s)\|_{H^{3}} d s \\
& \leqq C \int_{t}^{+\infty}(1+s)^{-\frac{p-1}{3}\left(1-\frac{2}{q}\right)} d s=C \int_{t}^{+\infty}(1+s)^{-\frac{(p-1)^{2}}{3 p}} d s \rightarrow 0,
\end{aligned}
$$

as $t \rightarrow+\infty$, for $p>5 / 2+\sqrt{21} / 2$. The proof of the theorem is then complete.

Acknowledgment. This project was partially supported by the National Natural Sciences Foundation of China.

\section{References}

1. Abdelouhab, L., Bona, J.L., Felland, M., Saut, J.C.: Physica D 40, 360-392 (1989)

2. Case, K.M.: Properties of the Benjamin-Ono equation. J. Math. Phys. 20, 972-977 (1979)

3. Ginibre, J., Tsutsumi, Y.: Uniqueness of solutions for the generalized KdV equation. SIAM J. Math. Anal 20, (6), 1388-1425 (1989)

4. Guo Boling, Tan Shaobin.: On smooth solution to the initial value problem for the mixed nonlinear Schrodinger equations. Proc. Roy. Soc. Edinburgh 119A, 31-45 (1991)

5. Joseph, R.I.: Solitary waves in a finite depth fluid. J. Phys. A, 10, L225-L227 (1977)

6. Kenig, C.E., Ponce, G., Vega, L.: On the generalized KdV equation. Duke Math. J, 59, (3), 585-610 (1989)

7. Klainerman, S., Ponce, G.: Global small amplitude solution to non-linear evolution equations. Commun. Pure Appl. Math.. 37, 133-141 (1983)

8. Iorio, R.: On the Cauchy problem for the Benjamin-Ono equation. Comm. PDE 11, 1031-1081 (1986)

9. Ponce, G.: Smoothing properties of solutions to the Benjamin-Ono equation. Diff. and Integral Equations 4, (3), 527-542 (1991)

10. Satsuma, J., Ablowitz, M.J., Kodama, Y.: On an internal Wave equation describing a stratified fluid with finite depth. Phys. Lett. A 73, 283-286 (1979)

11. Shatah, J.: Global existence of small solutions to nonlinear evolution equations. J. Diff. Eqs. 46, 609-625 (1982) 
12. Sidi, A., Sulem, C., Sulem, P.L.: On the long time behavior of a generalized KdV equation. Acta Appl. Math. 7, 35-47 (1986)

13. Stein, E.M.: Singular integrals and differentiability properties of functions. Princeton, NJ: Princeton University Press, 1970

14. Stein, E.M.: Oscillatory integrals in Fourier analysis. Beijing Lecture in Harmonic Analysis. Princeton, NJ: Princeton University Press, 1986, pp. 307-355

15. Strauss, W.A.: Nonlinear scattering theory of low energy. J. Funct. Anal. 41, 110-133 (1981)

16. Zhou Yulin, Guo Boling,: Initial value problem for a nonlinear singular Integral-Differential equation of deep water. Lecture Notes in Math. 1306, pp. 278-290

17. Zhou Yulin, Guo Boling, Tan Shaobin,: On the Cauchy problem for the equation of finite-depth fluids. J. PDE 5, (1), 1-16 (1992)

Communicated by H. Araki 
